\title{
La brecha de género en el deporte:
}

\section{El caso de una marginación histórica y socialmente consentida}

\author{
The gender gap in sport:
}

The case of a historically and socially accepted marginalization

Lluisa Aitana Sauleda Martínez ${ }^{1}$, Diego Gavilán Martín² y Jenny Martínez Benítez ${ }^{3}$

${ }^{1}$ Graduada en Educación Infantil por la Universidad de Alicante. Máster en Investigación

Educativa. Becaria por la Universidad de Alicante. E-mail: lasm@alu.ua.es

${ }^{2}$ Doctorando de Investigación Educativa por la Universidad de Alicante.

E-mail: diego.gavilan@alu.ua.es

${ }^{3}$ Doctora en Investigación Educativa por la Universidad de Alicante. Profesora Titular de la

Universidad Central de Ecuador. E-mail: jemartinez@uce.edu.ec

Universidad de Alicante. Alicante, España.

Universidad Central de Ecuador. Quito, Ecuador.

\section{Resumen}

El deporte se considera un medio esencial para favorecer la salud y el desarrollo de los ciudadanos. A su vez, es el camino para alcanzar otras metas como la paz, la tolerancia, el respeto, la democracia o la inclusión de los grupos marginados, que es el caso de las mujeres y niñas. Sin embargo, existe muy poco reconocimiento de la marginación de género en el mundo del deporte. Este estudio, a través de informes y datos estadísticos, analiza cómo la mujer es relegada de los cargos directivos y de los espacios de toma de decisiones y, asimismo, cómo es excluida del cuerpo de entrenadores, asesores y jurados deportivos, lo cual le quita toda visibilidad. Si la mayoría de los estudios vinculados con el deporte es ocupado por los varones y su índice de especialización en masters y doctorados es consecuentemente más alto en la mayoría de países, debemos prever que, desafortunadamente, la brecha no se está cerrando y la proyección de los varones en las ocupaciones laborales vinculadas al mundo deportivo continuará perpetuando espacios deportivos masculinizados, en los que el capital humano y la valiosa aportación de las mujeres se seguirá perdiendo. El análisis realizado muestra, a diversos niveles, la permanencia de la brecha de género en el deporte.

Palabras clave: actividad física y deportiva, diferenciales de género, espacio masculinizado.

\section{Abstract}

First of all, we must consider that the World Health Organization -WHO Global Action Plan on Physical Activity 2018-2030 Report (2018) points out the scarce physical activity implemented by citizens, and especially how this deficiency is focused on women. Then, it is important to analyze all aspects connected with this disaffection that it takes women away from the health benefits of sports and physical activity. We also must analyze the powerful effort of the international institutions to alert about the gender gap. And why have these reports not been enough listened to, or effective? The review of specific data as the presence of women in the Olympic Games, the 
women absence in responsibilities, decisions and management positions of the sportive field, or the differentials in the quarry of highlevel athletes confirm in almost all levels and scopes the existence and overlooked gender gap. The alert of international organizations, on a continuous basis, has not made a dent in the marginalization of gender in the sport field (United Nations Women - UNWomen, 2007, 2016 \& 2019). Girls and women have fewer opportunities and support when they participate in sport activities. And in the event that they manage to become professional athletes, they find another important gap such as salary. Likewise, the fact that women are scarcely present in Higher Education grades related to physical activity and sports is a significant indication of how little the situation can change. If the majority of studies associated to sport are occupied by men, and their specialization rate in masters and doctorates is consequently higher, we must anticipate that, unfortunately, the gap is not closing and the projection of men in the work occupations related to the sport world will continue to perpetuate masculinized sports spaces where human capital and the valuable contribution of women will continue to be lost. It is essential to sensitize future teachers to the importance of not leaving women behind in this traditionally paternalized, patronalized and pastoralized sport field. For this reason, we consider that the curricular integration of Sustainable Development Goals (SDGs) (UN, 2015), specifically SDG 5- Gender equality in sport studies could be a relevant way to reach awareness in the reduction of gender differentials in sports. The international agenda agreed in the SDGs commits demands us to change the way in which we think and act in favor of people, peace, prosperity, the environment and equity. Along these lines, we come to three questions that need to be reflected on and answered as a social collective: Why hasn't the expansion of sport spread by the same percentage to girls and women? Where and why, on the way to an equitable sport fabric, have we lost women?
And what commitment do we have as citizens with gender equality in sports? Undoubtedly, the lack of insertion opportunities in communities of initiates and of support in mentors, the fear of family conciliation, and other complex factors centered on gender stereotypes makes women athletes generally accept the space that the sports community assigns them. The underrepresentation of women in the national and international sports network demands from women themselves greater awareness and greater resistance to exclusion. As long as social contexts and cultural practices as a whole do not change, women athletes will have to fight hard to lead the issues of inequality of opportunities in the sports world.

Keywords: sport and physic activity, gender differential.

\section{Introducción}

La ausencia de actividad física y deportiva es considerada como un factor de riesgo para la aparición y el desarrollo de enfermedades (Yaffe y Gough, 2013). La propensión al sobrepeso de gran parte de la población, que incluye a los jóvenes, constituye un problema de orden prioritario. Asimismo, recientes descubrimientos señalan cómo los efectos de la obesidad afectan las funciones cognitivas de forma negativa (Erion et al., 2014; Himes y Reynolds, 2012). En relación con los beneficios psicológicos y sociales, se ha demostrado que la práctica físico-deportiva aumenta la autoestima (Ekeland, Heian, Hagen y Coren, 2005) y desarrolla competencias sociales, como la capacidad de afrontar situaciones o la tenacidad en la persecución de metas, lo cual crea capacitaciones beneficiosas en quien la realiza. Así, la práctica deportiva ayuda a desarrollar la constancia, la tenacidad y la focalización en el reto (Cerda, Saiz y Vergara, 2018). De igual forma, puede ayudar a los adolescentes a reducir el estrés y a mejorar su estilo de afrontamiento, ya que la actividad ejercitada y el apoyo en el equipo aminoran la agresividad y el exceso de energía que pueden 
tener aquellos que son sedentarios (Guadarrama, Mendoza, Márquez, Veytia y Martín, 2018; Pangrazi, 2000). Por el contrario, el sedentarismo ha sido asociado con estados depresivos y problemas de salud (Biddle y Asare, 2011). Además de la pérdida de efectos saludables que produce la insuficiencia de la práctica física y deportiva, hay otra pérdida de oportunidades de capacitación personal y social similar al producido por la brecha digital en las poblaciones desfavorecidas (Lopera y Echevarría, 2018). Numerosos estudios advierten que los niveles de actividad física tienden a disminuir con la edad y esa disminución, tal como señalan Telama y Yang (2000), así como Michaud, Jeannin y Suris (2006), es marcadamente más prevalente en la población adolescente femenina por razones culturales, sociales y económicas ya conocidas, lo que supone otra pérdida de oportunidades más para las mujeres.

Por ello, existe la necesidad de fomentar la práctica del deporte con el objetivo de mejorar la salud física y mental, así como la calidad de vida en la población. En el campo de las mujeres, los organismos internacionales nos alertan de que su situación en relación con la pobreza y la educación es más compleja que en los varones (Organización de Naciones Unidas [ONU], 2018; Organización de las Naciones Unidas para la Educación, la Ciencia y la Cultura [UNESCO], 2017; UNESCO 2019). En la actividad física y deportiva, también son las grandes excluidas, según todos los informes internacionales como el de United Nations Women (UNWomen, 2019).

La realidad estadística sobre las diferencias de género se observa en todos los ámbitos y en especial en el mundo deportivo, y las políticas de género más igualitarias no suelen hacerse efectivas en la práctica. El deporte, en teoría, tiene el poder de trascender las barreras de sexo, raza, religión y nacionalidad y es una actividad muy relevante para alcanzar la salud física y psíquica que el ser humano necesita (UNWomen, 2016). Pero en el caso de desafiar los estereotipos de género, no podemos negar que, a pesar de ello, la participación en estas actividades no ha sido equitativa entre mujeres y hombres. Además, cabe destacar que en algunas disciplinas deportivas la incorporación de la mujer ha sido muy tardía, por ejemplo, en el atletismo o el judo, lo cual sitúa a la mujer en una posición de desigualdad respecto del hombre (Iglesias, Lozano y Manchado, 2013). Aun así, el deporte influye de manera positiva en la equidad de género y en la construcción de un mundo mejor ya que, al practicar deporte, la mujer puede beneficiarse desarrollando nuevas habilidades $\mathrm{y}$ alcanzando la confianza para sobrevivir en la sociedad actual (De Varona, 2018). Con la finalidad de aportar una pequeña contribución a la sensibilización en esta significativa brecha, el estudio se centra en cuestiones de análisis de cuatro casos relevantes para visualizar los diferenciales en el campo deportivo:

- Esfuerzos institucionales internacionales para alertar la brecha de género.

- La presencia de la mujer en los juegos olímpicos.

- La ausencia de equidad en las responsabilidades, decisiones y cargos directivos.

- Los diferenciales en la cantera de los deportistas de alto nivel.

\section{Análisis de los esfuerzos}

\section{institucionales en la historia de la}

\section{desigualdad en el deporte}

Con el fin de paliar esta grave problemática, la medicina preventiva y los gobiernos realizan un gran esfuerzo de enculturación acerca de las ventajas de la práctica deportiva (Atkin, Gorely, Biddle, Cavil y Foster, 2011). Las organizaciones intergubernamentales coinciden en situar los deportes en el camino hacia el desarrollo humano, social, económico y político. No obstante, estudios realizados por la Organización Mundial de la Salud (OMS) (2010) alertan que el porcentaje de actividad 
física va a reducirse en un $10 \%$ en el año 2025. Cabe señalar que este fenómeno se ha producido con mayor intensidad en países con rentas e ingresos menores, por ejemplo, en el este de Europa o Latinoamérica. Guthold, Stevens, Riley y Bull (2018) realizaron una investigación sobre el informe $W H O$ Global Action Plan on Physical Activity 2018-2030 (WHO, 2018) publicado en The Lancet Global Health Journal, uno de los más prestigiosos focos de investigaciones en salud mundial. La investigación concluye que el nivel de actividad física sigue siendo insuficiente en un cuarto de la población adulta, con los datos recabados en 168 países. Por un lado, existen altos niveles de inactividad en los países con rentas bajas, debido a la falta de recursos y cultura física, como es el caso de Latinoamérica. Por otro lado, en países de altos ingresos, el aumento del sedentarismo debido al tipo de puestos de empleo y de desplazamiento hace que también aumente la inactividad física. En ambos casos, esta inactividad es mayor en el caso de las mujeres, que encuentran mayores barreras para la actividad física. Uno de los motivos es el que hace referencia a la falta de tiempo para el ocio entre el sector femenino, ya que su disponibilidad está centrada en las tareas domésticas, el cuidado de personas e incluso en el trabajo remunerado fuera de casa. Esto no ocurre en el caso del género masculino, el cual, tras el trabajo, dispone de tiempo libre para dedicar al deporte. Por ello, para reducir y evitar la desigualdad de género, los cambios en la cultura, los roles tradicionales y el apoyo social para la mujer, facilitarán el aumento en la práctica del deporte por parte del género femenino.

Al analizar la historia, se observa la débil posición de la mujer en diversos ámbitos, como en lo social, político, legal, económico, educativo y físico. En la evolución de la historia que ha protagonizado la mujer en el mundo del deporte, se han producido algunos logros en lo que se refiere a su repercusión, pero también discriminación y división. En lo que se refiere al mundo del deporte, a la mujer no solo no se le ha dado la oportunidad de mostrar todas sus potencialidades sino que el contexto masculinizado la ha relegado a un tercer plano (Hanson, 2012). La práctica del deporte de hombres y mujeres siempre se ha visto desde puntos de vista diferentes. En el caso de la mujer, se la consideraba como un ser débil para el deporte $\mathrm{y}$, a su vez, se pensaba que el deporte sería dañino para la salud de la mujer, sobre todo para su cuerpo en su función reproductiva (Diez, 1996). Sus actividades principales se centraban en el cuidado de personas o en tareas domésticas, las cuales no eran consideradas, ni social ni económicamente, como actividades productivas. Como ocurre en el caso de los hombres, las actividades fuera de casa se consideraban como trabajo, lo que conllevaba a un merecido tiempo libre para practicar deporte. Además, la mujer se enfrenta a diversas restricciones y otros peligros que le dificultan su participación en el deporte y a eso se suma la ausencia de modelos femeninos, ya sean deportistas o coachers (Azurmendi, 2016), o aquellas que trabajan en puestos de responsabilidad en instituciones deportivas; todo ello ha sido también un factor determinante (Sever, 2008).

En beneficio de la mujer, en la sociedad industrial del siglo XVIII, los movimientos feministas adoptaron estrategias para conseguir la equidad de género considerando los roles, las perspectivas y las responsabilidades tanto de mujeres como de hombres (Meier, 2005). Una de las medidas para alcanzar ese objetivo era utilizar el deporte, ya que no solo posee un gran potencial para mejorar la salud física y mental, sino que además se considera como un medio capaz de abrir las puertas a la mujer, permitiéndole beneficiarse positivamente de él y eliminar barreras para la completa participación de cada género en la sociedad como la Organización para la Cooperación y el Desarrollo Económicos-OCDE (2014) recomienda. Respecto a los logros iniciales, sirvan de ejemplo los casos de Helen Madison, como la primera mujer en nadar las 100 yardas en un minuto en 1932, 
o Nawal El Moutawakel de Marruecos, la primera mujer en ganar una medalla olímpica por los 400 metros en carrera de obstáculos (ONU, 2006). Entre las primeras declaraciones internacionales, se destaca la Declaración Maggling en 2003 (Sever, 2008), fundada en el principio del deporte como un derecho que incluía medidas para introducir a la mujer en iniciativas deportivas, además de promover la necesidad de eliminar estereotipos (Hardmean y Marshall, 2005). Sin embargo, a pesar de muchas y posteriores recomendaciones, no existe una clara evidencia que demuestre el impacto del deporte femenino en la brecha de género. Entre otros factores, porque en el mundo existen diversas culturas del deporte, que son la causa de las diferencias de género. Por ejemplo, en países del Mediterráneo, en los que predominan las sociedades patriarcales, es la mujer la que practica menos deporte que el hombre (Capranica, Piacentini, Halson, Myburgh, Ogasawara, Millard-Staffors, 2013).

La ONU ha luchado denodadamente por erradicar los diferenciales de género $y$ ha prestado especial atención a la participación de la mujer en el deporte. El informe de la Organización de las Naciones Unidas para la Educación, la Ciencia y la Cultura (UNESCO, 2004) así como la Asamblea General de las Naciones Unidas (ONU, 2006) incluyen el deporte como medio para promover la educación, la salud y la paz, así como la necesidad de promover y aumentar la presencia de la mujer en el mundo deportivo a través de políticas educativas.

Ya en 2007, UNWomen presentó el informe Women 2000 and beyond, con especial desarrollo del tema: mujeres, igualdad de género y deporte. En 2014, UNESCO presentó el World Wide Survey of School Physical Education, con una sección dedicada a cuestiones de equidad de género y discapacidad. En 2015, el Informe del desarrollo humano (Programa de las Naciones Unidas para el Desarrollo [PNUD], 2015) abogaba por el desarrollo de las capacidades humanas sin discrimina- ciones. En este sentido, Lopera y Echeverri (2018) subrayan que privar del potencial humano a un grupo social es inadmisible. La plataforma para el Deporte, el Desarrollo y la Paz (DDP, 2016) adoptó una resolución en diciembre de 2016, en la que reafirmaba el rol del deporte como medio de la promoción de la educación, la salud el desarrollo y la paz. La Asamblea General de las Naciones Unidas (2006), junto a los 50 países participantes, entre los que se encuentran únicamente cinco países latinoamericanos, decidió incluir en su agenda el tema deporte para el desarrollo y la paz. En este texto, la Asamblea General acentúa especialmente la necesidad de incrementar la presencia de la mujer y alcanzar la igualdad de género en el campo deportivo, especialmente a través de políticas educativas, necesidad que ya aparece en la Agenda 2030 para el Desarrollo Sostenible, que incluye la igualdad de la mujer (UNWomen, 2018).

El informe de la UNESCO Empowering girls and women through Physical Education and Sport, reportado por Kirk (2012) señala la importancia de los programas de Educación Física para jóvenes con el objetivo de desarrollar habilidades, conocimiento, autoestima, así como estilos de vida activos. Por otro lado, reconoce que la mujer se enfrenta a actitudes sexistas debido a las diferencias biológicas entre mujeres y hombres, por las cuales se les consideran inferiores, lo que limita su participación en el deporte. Otro aspecto que supone una barrera lo viven los estudiantes de Educación Física, la cual está enfocada en la enseñanza de técnicas sacadas del contexto del verdadero deporte y el juego, así como la trasmisión de valores como la competitividad en vez de la cooperación. En la misma línea, el grupo de expertos de la European Commission (2014a) emite las recomendaciones en equidad de género y deporte. Según el estudio especial del Eurobarometer 412 Deporte $\mathrm{y}$ actividad física (European Commission, 2014b), los hombres practican más deporte que las mujeres, especialmente en el grupo de edad 15-24. También las mujeres están 
infravaloradas en roles de liderazgo en el coaching, en los medios de comunicación y en la toma de decisiones. Por ello, un enfoque a favor de la igualdad puede evitar los estereotipos sexistas y ayudar a crear un entorno social positivo para todos.

El mencionado informe Igualdad de Género en Deporte. Propuesta de acciones estratégicas 2014-2020 (European Commission, 2014a) expone diversas estrategias como la igualdad de género en el coaching, el rol de los medios de comunicación contra los estereotipos y la lucha contra la violencia de género. Por ello, son necesarias estrategias favorables al género y directrices éticas que ayuden a desarrollar la educación y promuevan modelos femeninos para alentar a las niñas y las mujeres.

\section{Análisis de la presencia de la}

\section{mujer en los Juegos Olímpicos}

En la sociedad actual aún existe una gran desigualdad entre hombres y mujeres, y es por ello que la mujer debe hacer frente al modelo patriarcal (Ávalos, Martínez y Urrea, 2019). Esta desigualdad también conlleva una participación menor de las mujeres en las competiciones más importantes, como es el caso de los Juegos Olímpicos. Su fundador, Pierre de Coubertin, promovía el deporte como un espacio de hombres, sentía que la participación de la mujer era inapropiada ya que resumía sus funciones a las de adorno y protocolo, como meras espectadoras del deporte. Por lo tanto, el International Olympic Committee (IOC) fue considerado como un club de caballeros al que solo tenían acceso los hombres (Gallardo, González, Clemente, Santacruz y Espada, 2013). Adicionalmente, en 1894, las reglas de elegibilidad restringían la participación de la mujer en los Juegos Olímpicos debido a que el International Olympic Committee tuvo autoridad para implantar esta norma en los primeros Juegos modernos (IOC, 2019). Posteriormente, el Movimiento Olímpico afirmó que las reglas debían ser modificadas para evitar cualquier tipo de discriminación por razón de género, raza, religión, etcétera, (Teetzel, 2011) y produjo los primeros cambios en 1896. Sin embargo, en los primeros Juegos Olímpicos, en 1896, no participó ninguna mujer y no fue hasta 1900 , en los Juegos Olímpicos de París, cuando se aceptó a la mujer como deportista por primera vez. De 997 participantes, 22 mujeres tomaron parte en cinco deportes: golf, tenis, croquet, navegación y equitación. Fue en ese mismo año que Charlotte Cooper ganó la primera medalla de oro en tenis. Además, comenzaron a competir en eventos mixtos. Posteriormente en 1976, la participación de la mujer aumentó un $20 \%$, concretamente en los Juegos de Montreal (Keng, 2016).

A pesar de las iniciativas del IOC dirigidas a aumentar la representación femenina, cabe destacar que, en el año 2001, el porcentaje de mujeres era del $8.7 \%$ y no del $10 \%$ como se tenía previsto. Sin embargo, no se produjo un equilibrio en la participación de ambos sexos hasta el año 2012, en los Juegos Olímpicos de Londres. En ellos, no solo se incrementó el número de atletas femeninas, sino que había mujeres que competían en cada uno de los deportes del programa (Donelly, 2013). A pesar de los logros alcanzados, si lo comparamos con juegos anteriores, el número de eventos masculinos fue superior al de eventos femeninos. Además, de los 48 eventos exclusivos para un solo género, cabe destacar que 39 eran exclusivos para hombres y solo nueve para el género femenino.

Respecto a las mujeres españolas, también fue en los Juegos Olímpicos de París cuando aparecieron las primeras participantes. Sin embargo, tras ese momento, pasaron 30 años hasta que la mujer volvió a los juegos. Respecto a los Juegos Olímpicos de España, en 1992, cabe señalar que supusieron un progreso en el deporte femenino, ya que el número de participantes españolas era mayor que en los eventos anteriores y fue cuando se ganaron las primeras medallas. No obstante, ese año la representación femenina española tampoco 
fue elevada (Jiménez, 2015). Posteriormente, en los Juegos Olímpicos de Londres 2012 se produjeron importantes avances, por ejemplo, cuando la delegación femenina superó a la masculina al conseguir un número mayor de medallas. Más tarde, en el 2016, la participación de la mujer en el deporte se vio aumentada: alcanzó un $45 \%$ en los juegos de Río de Janeiro. A partir de estas experiencias, se prevé una participación del $48.8 \%$ de la mujer en los próximos Juegos Olímpicos de Tokio en 2020, el nivel más alto de equidad entre mujeres y hombres de la historia. ${ }^{1}$

\section{La brecha en cargos directivos: el estudio de caso del International Olympic Committee y el Comité Olímpico Español}

Ya que el deporte es uno de los elementos dedicados a promover la igualdad de género, el IOC tiene la responsabilidad de desarrollar acciones para apoyar y promocionar la participación de las mujeres en el deporte en todos los niveles (IOC, 2018), así como conseguir un equilibrio en el número de deportistas que participan en los juegos olímpicos. Para esto, debe desarrollar iniciativas que permitan el acceso de la mujer al deporte no solo como deportistas, sino en puestos de altos cargos y de administración. Aunque el número de mujeres deportistas ha aumentado en los últimos años, la participación del género femenino en la administración y en cargos directivos del IOC se mantiene en niveles bajos.

Respecto a la conformación del IOC, en 1900, Flor Isava Fonseca fue la primera mujer Tabla 1 elegida para formar parte de la junta directiva. Además, cabe destacar que de 2016 a 2017 se produjo un aumento en el número de miembros de género femenino, hasta alcanzar un total de cuatro mujeres entre los quince miembros que componen la junta $(27 \%)$. En referencia a los puestos directivos, en 2018 se produjo un aumento del número de mujeres como directoras de Junta. En los puestos de alta gerencia, se puede observar que, mientras en países como Suiza aumenta el número de mujeres, en España no se perciben los incrementos. Además, según una encuesta del IOC de 2015, solo el $8 \%$ de las mujeres ocupa el cargo de presidente de los diversos comités olímpicos. Respecto a los National Olympic Committees (NOCs), datos extraídos del programa IOC's People Management 2020 señalan que, en 2018, solo 13 mujeres llegaron a alcanzar el cargo de presidenta, mientras que 33 se mantuvieron como secretarias generales. Algo parecido ocurría en las federaciones internacionales, en las que solo cuatro federaciones tenían presidenta de género femenino (IOC, 2019).

El Comité Olímpico Español (COE), desde su fundación en 1912, ha vivido numerosos cambios con éxitos organizativos y deportivos. Pero respecto a los méritos alcanzados por la mujer, como es la consecución de puestos de responsabilidad en esta institución, no ha habido tanto éxito. En España, según los estudios de Méndez (2015), aunque la proporción de mujeres encargadas de la dirección y gestión de entidades deportivas ha aumentado, existe aún una predominancia del género masculino (ver Tabla 1).

Puestos de responsabilidad y distinciones en el Comité Olímpico Español.

\begin{tabular}{c|c|c|c|c} 
& Hombres & Mujeres & $\begin{array}{c}\text { FA\% } \\
\text { Hombres }\end{array}$ & $\begin{array}{c}\text { FA\% } \\
\text { Mujeres }\end{array}$ \\
\hline Asamblea General & 105 & 15 & 87.5 & 12.5 \\
Comité Ejecutivo & 19 & 5 & 79.1 & 20.8
\end{tabular}

${ }^{1} N$ del E.: A causa de la pandemia del COVID-19, los Juegos Olímpicos de Tokio 2020 fueron suspendidos y postergados para julio 2021. 
Sauleda Martínez, Gavilán Martín y Martínez Benítez

\begin{tabular}{c|c|c|c|c} 
& Hombres & Mujeres & $\begin{array}{c}\text { FA\% } \\
\text { Hombres }\end{array}$ & $\begin{array}{c}\text { FA\% } \\
\text { Mujeres }\end{array}$ \\
\hline Comisiones del COE & 198 & 58 & 77.3 & 22.6 \\
Distinciones & 1130 & 419 & 72.9 & 27.04
\end{tabular}

En la Tabla 1 se recogen los datos del COE (2019) y se puede observar claramente la gran diferencia entre sexos en relación con los diversos cargos que ocupan el COE, así como en la concesión de distinciones. Asimismo, se debe destacar que, del reducido número de mujeres, algunas se repiten debido a que ocupan además cargos honoríficos. Por ejemplo, la misma mujer aparece en la Asamblea General y en el Comité Ejecutivo; y la secretaria general es la misma persona para todas las diversas comisiones. Asimismo, cabe señalar que en algunos comités como el Comité de Ética hay una sola mujer de un grupo de ocho miembros, al igual que ocurre en la Comisión de Cultura y Educación Olímpica, con solo cuatro mujeres y 23 hombres, y en el Comité de Solidaridad, con una mujer y 15 hombres. Únicamente la Comisión de Mujer e Igualdad de Género está constituida por mujeres; en el resto predominan los miembros del sexo opuesto. Respecto a las distinciones, cabe destacar la Orden Olímpica de Oro, en la que las dos únicas mujeres que forman parte han sido nombradas por protocolo. Asimismo, en referencia a las medallas, es reducido el número de mujeres que las han ganado. Por tanto, se puede concluir que todavía existe un largo camino por recorrer antes de ver una igualdad total en el mundo del deporte. Las niñas y mujeres poseen menos oportunidades y seguridad cuando participan en actividades deportivas y, en el caso de que logren llegar a ser deportistas profesionales, se encuentran con otra brecha importante como es la salarial y que existe, por ejemplo, en el mundo del fútbol femenino.

\section{Análisis de caso: Entrenadores y}

\section{presidentes}

Los deportes colectivos pretenden fomentar el valor del trabajo en equipo, la solidaridad y la planificación colaborativa, al tiempo que exaltan los méritos y la competición entre iguales. Pero en la realidad esto no ocurre así. El Comité Nacional de Fútbol Femenino según el Medio Oficial de la Real Federación Española de Fútbol (RFEF, 2019a) -el cual se encarga de organizar y promover el fútbol practicado por mujeres- se caracteriza por poseer un mayor número de cargos de responsabilidad y de entrenamiento asignados a hombres. Concretamente, forman parte de su plantilla el doble de hombres que de mujeres (22 hombres y 11 mujeres); y es destacable, además, que el presidente del comité es varón. Respecto al Comité de Entrenadores, tanto en los distintos comités territoriales como en la junta directiva de dicha federación, los cargos de responsabilidad son ocupados por hombres (RFEF, 2019b).

Con relación al personal directivo de las federaciones españolas, datos recogidos por el Ministerio de Cultura y Deporte (2019) muestran que existe una predominancia de los hombres sobre las mujeres. De las 68 federaciones deportivas, en las escasas ocasiones en las que una mujer es miembro del equipo directivo, esta ocupa el puesto de secretaria, no de presidenta: por ejemplo, en judo, bádminton o atletismo. Únicamente hay excepciones en la federación de vela y remo, en las cuales una mujer tiene el puesto de presidenta, así como natación y tiro con arco, con una mujer en gerencia y una directora técnica en gimnasia. Con ello se observa, pues, la existencia de espacios masculinizados 
ya que en los deportes masculinos son los deportistas de élite los miembros de la junta e incluso los periodistas, y no hay ningún tipo de presencia femenina, mientras que sí hay presencia masculina en los deportes femeninos. En suma, el ámbito deportivo sigue dominado por el género masculino y eso no solo limita el acceso de la mujer, sino que en muchos casos la excluye de su participación (Gallardo et al., 2013).

La participación femenina en puestos de responsabilidad en organizaciones y asociaciones deportivas se ha asociado frecuentemente a la disponibilidad de tiempo, flexibilidad y energía, aduciendo que, en el caso de la mujer, su situación familiar hace que esta disponibilidad sea escasa respecto al hombre. Esta postura es una simple excusa para dejar la toma de decisiones en manos de los hombres (Selva, Pallarès y González, 2012).

Sin embargo, desde 1991 se empezó a requerir que todos los deportes que se añadieran en el programa olímpico incluyeran eventos femeninos como medio para aumentar la implicación de la mujer en el mundo deportivo y en los juegos olímpicos en el cual participaban tanto el IOC como la Federación Internacional de Deporte. A pesar de esto, en la labor de entrenadoras, las mujeres seguían teniendo una baja representación. Como muestran las estadísticas sobre la mujer en los Juegos Olímpicos (IOC, 2019), desde aquellos celebrados en el 2010 hasta los del 2016 solo ha habido un aumento del $1 \%$ de la mujer entrenadora. Por tanto, no solo el número de entrenadoras es menor que el de entrenadores, sino que además las mujeres permanecen menos tiempo entrenando a diferencia del hombre, el cual tiene una dedicación completa. La mayor parte de entrenadoras tienen experiencia previa como deportistas y han participado en competiciones de carácter territorial, nacional e internacional. Por otra parte, aunque hay más mujeres que hombres que poseen un mayor nivel de estudios en los puestos de entrenador o entrenadora, el porcentaje de ellos es mayor conforme avanza el nivel deportivo (Pfister, 2013).

Respecto al estilo de entrenamiento, las entrenadoras utilizan un estilo diferente al de los hombres; las mujeres tienden a centrarse en el proceso de entrenamiento y en la calidad de las relaciones sociales, fomentando valores de cooperación, empatía y comunicación. Los hombres, sin embargo, desarrollan un estilo de liderazgo centrado en los logros y resultados, adoptando una actitud más competitiva y autoritaria.

En la Tabla 2 se observan los datos del informe Gendery Coaching Report Card: London 2012 Olympics (International Council for Coaching Excellence, 2014) que muestra los porcentajes de mujeres entrenadoras por continente, destacando que en Europa solo hubo un $11 \%$ de mujeres frente a un $89 \%$ de hombres en los Juegos Olímpicos de 2012. Más concretamente, en España, la cantidad de entrenadoras en el mismo evento es de siete mujeres y 59 hombres. La llamada del International Council for Coaching Excellence (2014) hacia la igualdad como único medio de alcanzar la excelencia no debe ser desconsiderada.

Tabla 2

Número total de entrenadores acreditados en los Juegos Olímpicos de 2012.

\begin{tabular}{c|c|c}
\hline $\begin{array}{c}\text { Número total de entrenadores } \\
\text { acreditados }\end{array}$ & $\begin{array}{c}\text { Número de entrenadores } \\
\text { masculinos acreditados }\end{array}$ & $\begin{array}{c}\text { Número de entrenadoras } \\
\text { femeninas acreditadas }\end{array}$ \\
3225 & $2867(89 \%)$ & $358(11 \%)$
\end{tabular}




\section{Análisis del caso de los deportistas}

\section{de alto nivel y de alto rendimiento}

\section{en España}

Los diferenciales de género en deportistas de élite y en el deporte profesional se detectan ampliamente en los informes. Por ejemplo, el informe Plan de Promoción de la igualdad entre mujeres y hombres en el sector deportivo (Gobierno Vasco, 2013) señala la necesidad de incentivar el apoyo a las deportistas en todos los ámbitos, ya que en el deporte profesional se detectan espacios en los que la presencia femenina se constituye en ausencia. Un aspecto remarcable es que las deportistas presentan mayor preocupación por las ayudas económicas y la inserción laboral que los varones, pues las estadísticas las muestran en inferioridad de condiciones en ambos aspectos frente a sus compañeros varones. Las diferencias porcentuales de género entre entrenadores, árbitros y jueces les niegan esas posibles salidas profesionales. Además, los recursos públicos en subvenciones son frecuentemente monopolizados por los deportes masculinos. Las deportistas, al tener muy restringida la participación en circuitos profesionales, resultan más perjudicadas en los espacios federativos.

La Tabla 3 muestra los datos obtenidos de la Resolución de la Presidencia del Consejo Superior de Deportes, del Ministerio de Cultura y Deporte (Consejo Superior de Deportes, 2019).

\section{Tabla 3}

Relación de los deportistas de alto nivel y alto rendimiento en España.

Número total de deportistas de alto nivel y de alto rendimiento

486

Como se aprecia en la tabla, la cartera de deportistas de alto nivel en España ofrece pocas posibilidades de nivelar su futuro profesional. Como reclama el informe del Gobierno Vasco mencionado, los esfuerzos por conseguir igualdad de oportunidades en el deporte deben articularse en todos sus ámbitos, desde la actividad física y deportiva en todos los niveles del sistema educativo hasta el deporte federado y el de alto rendimiento, atendiendo la oportunidad de acceso y la presencia y permanencia de las mujeres en los 18 puestos de responsabilidad y toma de decisiones en el ámbito del deporte. El hecho de la escasa presencia de las mujeres en la formación académica universitaria relacionada con la actividad física y deportiva es un indicio significativo de lo poco que puede cambiar la situación. Si la mayoría de los estudios vinculados con el deporte es ocupado por
Número de deportistas varones

318

los varones y su índice de especialización en masters y doctorados es consecuentemente más alto, debemos prever que, desafortunadamente, la brecha no se está cerrando y la proyección de los varones en las ocupaciones laborales vinculadas al mundo deportivo continuará perpetuando espacios deportivos masculinizados en los que el capital humano y el valioso aporte de las mujeres se seguirá perdiendo.

\section{Conclusión}

El deporte ha sido un ámbito tradicionalmente masculino al que las mujeres no solo no han tenido un acceso fácil, sino que tuvieron una participación limitada por los estereotipos y los roles asignados socialmente a cada sexo. A pesar de estos obstáculos, y si bien las mujeres han ido incorporándose y participando en un 
mayor número de deportes, el aumento de la práctica deportiva por parte de las mujeres no ha conllevado un aumento proporcional de la participación de las mujeres en los estamentos técnicos y directivos, en los que su presencia es claramente inferior a la de hombres. A la mujer se le exige más para demostrar que tiene las capacidades y habilidades suficientes para participar en el deporte y alcanzar sus metas profesionales. Se podría aplicar la historia de Alicia a través del espejo, de Lewis Carroll, en la que la mujer ha de correr dos veces más para llegar al mismo sitio que los varones. Y es que el deporte sigue siendo un espacio y un ámbito social en el que la identidad y hegemonía masculina se ha vuelto más resistente al cambio, y los medios de comunicación colaboran para que este hecho sea aceptado socialmente, privilegiando los deportes masculinos en la pantalla. Por esa razón, la discriminación y subordinación que se produce en las relaciones entre ambos géneros refleja la pervivencia de la organización patriarcal.

La educación es la llave que abre todas las puertas y el profesorado de Actividad Física y Deportiva tiene la relevante misión de abrir las mentes de sus estudiantes a esta problemática. Es necesario que el cambio se produzca desde los ámbitos educativo, social e institucional si se quiere una valoración social del deporte como un ámbito de equidad, democrático y ético.

\section{Referencias bibliográficas}

Atkin, A., Gorely, T., Biddle, S., Cavill, N. y Foster, C. (2011). Interventions to promote physical activity in young people conducted in the hours immediately after school: a systematic review. International Journal of Behavioral Medicine, 18, 176-187. https://doi.org/10.1007/s12529$\underline{010-9111-z}$

Ávalos, M. A., Martínez, M. A. y Urrea, M. E. (2019). Percepción del alumnado de Educación Física y de Educación Primaria sobre diferenciales de género en las aulas universitarias: Estudio de caso. Revista Complu- tense de Educación, 30, 847-862. https://doi. org/10.5209/RCED.59420

Azurmendi, A. (2016). Obstáculos psicosociales para la participación de las mujeres en el deporte como entrenadoras y árbitras. Universidad del País Vasco, Donostia. http://hdl. handle.net/10810/26195

Biddle, S. y Asare, M. (2011). Physical activity and mental health in children and adolescents: a review of reviews. British Journal of Sports Medicine, 45, 886-895. https://doi.org/10.1136/ bjsports-2011-090185

Capranica, L., Piacentini, M. F., Halson, H., Myburgh, K., Ogasawara, E. y Millard-Stafford, M. (2013). The gender gap in sport performance: Equity influences equality. International Journal of Sports Physiology and Performance, 8(1), 99-103. https://doi. org/10.1123/ijspp.8.1.99

Cerda, C., Saiz, J. L. y Vergara, D. (2018). Tenacidad en estudiantes universitarios chilenos: Un estudio inicial de su estructura y red nomológica. Interdisciplinaria, 35(2), 409-423. https:// doi.org/10.16888/interd.2021.38.1.1ISSN: 1668-7027

Comité Olímpico Español [COE]. (2019). Normas, buen gobierno y transparencia. http:// www.coe.es

Consejo Superior de Deportes. (2019). Personal directivo de las federaciones españolas. Madrid: Ministerio de Cultura y Deporte. www.csd. gob.es/es/federaciones-y-asociaciones/federaciones-deportivas-espanolas/personal-directivo-de-las-federaciones-espanolas

Deporte, Desarrollo y Paz -DDP (2016). La plataforma Deporte, el Desarrollo y la Paz de la ONU. https://deportedesarrolloypaz.org/el-deporte-para-el-desarrollo-y-la-paz/

De Varona, D. (2018). IOC is leading the way in gender equality. https://www.olympic.org

Diez, C. (1996). Deporte y construcción de las relaciones de género. Gazeta de Antropología, 12, 1-12. http://hdl.handle.net/10481/13591

Donelly, P. y Donelly, M. (2013). The London 2012 Olympics: A gender equality audit. Research Report University of Toronto. http:// www.sportpolicystudies.ca 
Ekeland, E., Heian, F., Hagen, K. y Coren, E. (2005). Can exercise improve self-esteem in children and young people? A systematic review of randomised controlled trials. British Journal of Sport Medicine, 39(11) 792-798. https://doi.org/10.1136/bjsm.2004.017707

Erion, J., Wosiski-Kuhn, M., Dey, A., Hao, S., Davis, C., Pollock, N. y Stranahan, A. (2014). Obesity elicits interleukin 1- Mediated deficits in hippocampal synaptic plasticity. The Journal of Neuroscience, 34(7), 2618-2631. https://doi. org/10.1523/JNEUROSCI.4200-13.2014

European Commission. (2014a). Gender Equality in Sport: Proposal for strategic actions 20142020. Brussels: Publication Office of European Commission. https://op.europa.eu/en/publication-detail/-/publication/7e7a7ff2-5bff-42b490f9-8266b3ddc01c

European Commission. (2014b). Special Eurobarometer 412 Sport and physical activity report. https://ec.europa.eu/commfrontoffice/publicopinion/archives/ebs/ebs_412_en.pdf

Gallardo, J., González, R. M., Clemente, A. L., Santacruz, J. A. y Espada, M. (2013). Los puestos de dirección en el ámbito deportivo: revisión de las teorías y estudios sobre el caso de la mujer. Revista Feminismo/s, 21, 91-115. http://hdl.handle.net/20.500.12020/657

Gallego, B. y Estebaranz, A. (2003). Mujeres que abren camino en el deporte: factores influyentes en las situaciones de igualdad $y$ discriminación. Universidad de Sevilla. https://docplayer.es/14284932-Mujeres-que-abren-camino-en-el-deporte-factores-influyentes-en-las-situaciones-de-igualdad-y-discriminacion.html o en http://www. mujerydeporte.org/documentos/docs/

Gobierno Vasco. (2013). Plan de promoción de la igualdad entre hombres y mujeres en el sector deportivo. Departamento de Educación, Política, Lingüística y Cultura. https://www. euskadi.eus/igualdad-deporte/web01-a2kirola/ es/

Guadarrama, R., Mendoza, V., Márquez, O., Veytia, M. y Martín, I. (2018). Diferencias en la aplicación de estrategias de afrontamiento adolescente entre alumnos hombres y mujeres del nivel medio superior. Interdisciplinaria, 35(1), 205-216. https://doi.org/10.16888/ interd.2018.35.1.11

Guthold, R., Stevens, G., Riley, L. M. y Bull, F. (2018). Worldwide trends in insufficient physical activity from 2001 to 2016: a pooled analysis of 358 population-based surveys with 1.9 million participants. The Lancet Global Health. $\quad$ https://doi.org/10.1016/S2214109X(18)30357-7

Hanson, V. (2012). The Inequality of Sport: Women $<$ Men. The Review: A Journal of Undergraduated Student Research, 13, 15-22. https://fisherpub.sjfc.edu/ur

Hardmean, K. y Marshall, J. (2005). Update on the state and status of Physical Education World-wide. En International Council of Sport Science and Physical Education. 2 World Summit on Physical Education. Magglingen, Switzerland., 2-3. https://www.icsspe.org/ content/2nd-world-summit-physical-education-0

Himes, C. y Reynolds, S. (2012). Effect of obesity on falls, injury, and disability. Journal of the American Geriatrics Society, 60, 124-129. $\quad$ https://doi.org/10.1111/j.15325415.2011.03767.x

Iglesias, M., Lozano, I. y Manchado, C. (2013). Deporte e igualdad: Las voces de las deportistas de élite. Feminismo/s, 21, 71-90. https:// doi.org/10.14198/fem.2013.21.05.

International Council for Coaching Excellence. (2014). Gender y Coaching Report Card: London 2012 Olympics. Leeds Metropolitan University. https://www.icce.ws/_assets/files/ norman.pdf

International Olympic Committee (IOC). (2016). Factsheet. Women in the Olympic Movement. Switherland: International Olympic Committee. https://stillmed.olympic.org/ media/Document\%20Library/OlympicOrg/ Factsheets-Reference-Documents/Women-inthe-Olympic-Movement/Factsheet-Women-inthe-Olympic-Movement.pdf

International Olympic Committee (IOC). (2019). Key dates in the history of women in the Olympic movement. https://www.olympic.org/ 
women-in-sport/background/key-datesI

International Olympic Committee (IOC) (2020). Statistics. Women at the Olympic Games. Switherland: International Olympic Committee. https://www.olympic.org/the-ioc

IOC's People Management (2020). IMD Governance Review at a glance. Summary of recommendations. https://stillmedab.olympic.org/ media/

Jiménez, R. (2015). El deporte femenino español en los Juegos Olímpicos. Madrid: Ministerio de Educación, Cultura y Deporte.

Keng, K. (2016). See 120 years of struggle for gender equality at the Olympics. PRI's The World. http://www.pri.org

Kirk, D. (2012). Empowering Girls and Women through Physical Education and Sport. Advocacy Brief. Bangkok: UNESCO Bangkok.

Lopera, I. y Echevarri, J. (2018). Libertad y desarrollo humano en las organizaciones. Interdisciplinaria, 35(2), 395-408. https:// doi.org/10.16888/interd.2018.35.2.9

Meier, M. (2005). Gender equity, sport and development. Working paper. Biel: Swiss Academy for Development. https://www.sportanddev. $\mathrm{org} / \mathrm{sites} /$ default/files/downloads/59__gender_ equity_sport_and_development.pdf

Méndez, B. (2015). El directivo deportivo del siglo XXI: Áreas de análisis. Revista Española de Educación Física y Deportes, 410, 41-55.

Michaud, P., Jeannin, A. y Suris, J. (2006). Correlates of extracurricular sport participation among Swiss adolescents. European Journal of Pediatrics, 165, 546-555. https:// doi.org/10.1007/s00431-006-0129-9

Ministerio de Cultura y Deporte. (2019). Resolución de 10 de junio de 2019, de la Presidencia del Consejo Superior de Deportes, sobre la relación de deportistas de alto nivel correspondiente al segundo listado del año 2018. https://www.boe.es/diario_boe/txt.php?id=BOE-A-2019-8875

Organización Mundial para la Salud (OMS). (2010). Recomendaciones mundiales sobre actividad física para la salud. Suiza: Organización Mundial para la Salud. https://www.who. int/dietphysicalactivity/factsheet_recommen- dations/es/

Organización de Naciones Unidas (ONU). (2006). Resolución 61/10 de la Asamblea General "El deporte como medio de promover la educación, la salud, el desarrollo y la paz". A/Res 61/10 (3 de noviembre de 2006). https://research. un.org/es/docs/ga/quick/regular/61

Organización de Naciones Unidas (ONU). (2016). ONU Mujeres Informe anual 20162017. Bruselas: ONU Mujeres. https://www. unwomen.org/es/digital-library/publications/2017/6/annual-report-2016-2017

Organización de Naciones Unidas (ONU). (2018). Informe de los objetivos de Desarrollo Sostenible. Nueva York: Naciones Unidas. http:// www.un.org/sustainabledevelopment/es/objetivos-de-desarrollo-sostenible

Organización para la Cooperación y el Desarrollo Económicos (OCDE). (2014). Social Institutions y Gender Index. France. https://www. oecd.org/dev/development-gender/press-release-sigi.htm

Pangrazi, R. P (2000). Promoting physical activity for youth. Journal of Science and Medicine in Sport, 3(3), 280-286. https://doi.org/10.1016/ S1440-2440(00)80037-7

Pfister, G. (2013). Outsiders: "Female coaches intruding upon a male domain?" En G. Pfister y M. K. Sisjord (Comps.), Gender and Sport .Changes and Challenges. Münster: Waxmann.

Programa de las Naciones Unidas para el Desarrollo (PNUD) (2015). Informe sobre Desarrollo humano. http://hdr.undp.org/sites/ default/files/2015_human_development_ report_overview_-_es.pdf

Real Federación Española de Fútbol. (2019a). Comité Nacional de Fútbol Femenino. https:// www.rfef.es/comites/comite-nacional-de-futbol-femenino

Real Federación Española de Fútbol (2019b). Comité de entrenadores. https://www.rfef.es/ noticias/entrenadores

Selva, C., Pallarès, S. y González, M. D. (2012). Una mirada a la conciliación a través de las mujeres deportistas. Revista de Psicología del Deporte, 2, 69-76. https:www.rpd-online.com/ article/view/v22-n1-selva-pallares-gonzalez 
Sever, C. (2005). Gender \& Sport. Mainstreaming gender in sports Projects. Bern: Swiss Agency for Development and Cooperation. https:// www.sportanddev.org/sites/default/files/downloads/9_gender_and_sport_mainstream_ gender_in_sports_projects.pdf

Teetzel, S. (2011). Rules and reform: eligibility, gender differences, and the Olympic Games. Sport in Society, 14, 386-398. https://doi.org/1 $\underline{0.1080 / 17430437.2011 .557275}$

Telama, R. y Yang, X. (2000). Decline of physical activity from youth to young adulthood in Finland. Medicine and Science in Sports and Exercise, 32, 1617-1622. https://doi. org/10.1097/00005768-200009000-00015

United Nations. (2015). The Sustainable Development Goals (SDGs). https://sdgs.un.org/es/ goals

United Nations Educational, Scientific and Cultural Organization (UNESCO). (2004). Fourth International Conference of ministers and senior officials responsible for Physical Education and Sport. Final Report. Greece: UNESCO https://unesdoc.unesco.org/ ark:/48223/pf0000140584

United Nations Educational, Scientific and Cultural Organization (UNESCO). (2014). World Wide Survey of School Physical Education. https:// en.unesco.org/inclusivepolicylab/e-teams/ quality-physical-education-qpe-policy-project/ documents/world-wide-survey-school-physical

United Nations Educational, Scientific and Cultural Organization (UNESCO). (2017). Education for Sustainable Development Goals. Learning objectives. France: United Nations Educational, Scientific and Cultural Organiza- tion. https://www.un.org/sustainabledevelopment/gender-equality/

United Nations Educational, Scientific and Cultural Organization (UNESCO). (2019). Atlas of gender inequality in education.http:// www.unesco.org

United Nations Women (UNWomen). (2007). Women, gender equality and sport. Women and Beyond 2000. https://www.unwomen. org/en/digital-library/publications/2007/12/ women2000-and-beyond-women-genderequality-and-sport

United Nations Women (UNWomen). (2016). In sport and for gender equality, One Win Leads to Another. https://asiapacific.unwomen.org/ en/news-and-events/stories/2016/08/in-sportand-for-gender-equality

United Nations Women (UNWomen). (2018). Agenda 2030 para el Desarrollo Sostenible, $\quad$ https://www.unwomen.org/es/ what-we-do/2030-agenda-for-sustainable-development

United NationsWomen (UNWomen). (2019). Women and Sport. Physical Education, Physical Activity and Sport, UNESCO $38^{\text {th }}$ General Conference. https://www.unwomen.org/en/ news/in-focus/women-and-sport

World Health Organization [WHO]. (2018). WHO Global Action Plan on Physical Activity 20182030. More active people for a healthier world. https://apps.who.int/iris/bitstream/handle/1066 5/272722/9789241514187-eng.pdf?ua=1

Yaffe, M. y Gough, N. (2013). 2012: Signaling breakthroughs of the year. Science Signal Journal, 6(256). $\quad$ https://doi.org/10.1126/ scisignal.2003881

Recibido: 24 de septiembre de 2019 Aceptado: 15 de marzo de 2021 